\title{
Systematic approach identifies RHOA as a potential biomarker therapeutic target for Asian gastric cancer
}

\author{
Hae Ryung Chang ${ }^{1,2, *}$, Seungyoon Nam ${ }^{1,3, *}$, Jinhyuk Lee ${ }^{4,5, *}$, Jin-Hee Kim ${ }^{6, *}$, Hae Rim \\ Jung ${ }^{1}$, Hee Seo Park ${ }^{7}$, Sungjin Park ${ }^{1,3}$, Young Zoo Ahn ${ }^{1}$, Iksoo Huh ${ }^{8}$, Curt Balch ${ }^{9}$, \\ Ja-Lok Ku${ }^{10}$, Garth Powis ${ }^{11}$, Taesung Park ${ }^{8}$, Jin-Hyun Jeong ${ }^{6}$, Yon Hui Kim ${ }^{1,12}$ \\ ${ }^{1}$ New Experimental Therapeutics Branch, National Cancer Center of Korea, Goyang-si, Republic of Korea \\ ${ }^{2}$ Research Institute of Women's Health, Sookmyung Women's University, Seoul, Republic of Korea \\ ${ }^{3}$ College of Medicine, Gachon University, Incheon, Republic of Korea \\ ${ }^{4}$ Korean Bioinformation Center, Korea Research Institute of Bioscience and Biotechnology, Daejeon, Republic of Korea \\ ${ }^{5}$ Department of Nanobiotechnology and Bioinformatics, Korea University of Science and Technology, Daejeon, Republic of \\ Korea \\ ${ }^{6}$ College of Pharmacy, Yonsei Institute of Pharmaceutical Sciences, Yonsei University, Incheon, Republic of Korea \\ ${ }^{7}$ Animal Sciences Branch, National Cancer Center of Korea, Goyang-si, Republic of Korea \\ ${ }^{8}$ Department of Statistics, Seoul National University, Seoul, Republic of Korea \\ ${ }^{9}$ Bioscience Advising, Ypsilanti, MI, USA \\ ${ }^{10}$ Korean Cell Line Bank, Seoul National University, Seoul, Republic of Korea \\ ${ }^{11}$ Cancer Center, Sanford-Burnham Prebys Medical Discovery Institute, La Jolla, CA, USA \\ ${ }^{12}$ Discovery Biology, CrystalGenomics Inc., Seongnam-si, Republic of Korea \\ *These authors have contributed equally to this work \\ Correspondence to: Yon Hui Kim, email: yhkim@cgxinc.com \\ Jin-Hyun Jeong, email: organicjeong@yonsei.ac.kr \\ Taesung Park, email: tspark@stats.snu.ac.kr \\ Keywords: gastric cancer, RHOA, G-protein, biomarker, therapeutic target \\ Received: July 11, $2016 \quad$ Accepted: October 17, $2016 \quad$ Published: October 28, 2016
}

\section{ABSTRACT}

Gastric cancer (GC) is a highly heterogeneous disease, in dire need of specific, biomarker-driven cancer therapies. While the accumulation of cancer "Big Data" has propelled the search for novel molecular targets for GC, its specific subpathway and cellular functions vary from patient to patient. In particular, mutations in the small GTPase gene RHOA have been identified in recent genome-wide sequencing of GC tumors. Moreover, protein overexpression of RHOA was reported in Chinese populations, while RHOA mutations were found in Caucasian GC tumors. To develop evidence-based precision medicine for heterogeneous cancers, we established a systematic approach to integrate transcriptomic and genomic data. Predicted signaling subpathways were then laboratory-validated both in vitro and in vivo, resulting in the identification of new candidate therapeutic targets. Here, we show: i) differences in RHOA expression patterns, and its pathway activity, between Asian and Caucasian GC tumors; ii) in vitro and in vivo perturbed RHOA expression inhibits GC cell growth in high RHOA-expressing cell lines; iii) inverse correlation between RHOA and RHOB expression; and iv) an innovative small molecule design strategy for RHOA inhibitors. In summary, RHOA, and its oncogenic signaling pathway, represent a strong biomarker-driven therapeutic target for Asian GC. This comprehensive strategy represents a promising approach for the development of "hit" compounds. 


\section{INTRODUCTION}

Gastric cancer (GC) is the fourth-most common cancer in the world, with an estimated 1.034 million new cases in 2015, and the third-highest cause of cancer deaths, estimated at 785,558 in 2014 [1]. GC mortality is highest in East Asia, with over half the world's total deaths, followed by Central and Eastern Europe, and Central and South America [1]. These statistics show clear disease differences based on geographic region, race, and ethnicity [1].

Surgery is the $1^{\text {st }}$ line treatment for GC, producing an overall survival rate of $60-70 \%$ for early stage disease (www.cancer.gov) [2]. High GC-incidence nations such as Japan and Korea now implement routine screening for early detection, when the disease is highly curable $[3,4]$. In many less developed countries, however, $\mathrm{GC}$ is mostly detected only in its advanced stages, precluding curative surgical resection and necessitating systematic treatment. The low efficacy of current therapies results in advanced or metastatic GC having a low survival rate of 5-20\%, and a particularly poor prognosis for peritoneal GC recurrence $[5,6]$. Together, these facts thus reinforce the urgent need for improved biomarker-driven, "targeted" therapeutic strategies.

A disease phenotype is the culmination of complex network interactions between multiple biological processes/pathways [7]. Consequently, "network medicine" can enhance the understanding of the detailed mechanisms and cellular progression of heterogeneous (e.g., gastric, pancreatic, etc.) cancers, revealing better biomarker-driven targets for drug development [7]. This approach, however, requires detailed analyses of multiple signaling transduction pathways, especially in specific tumor subpopulations. Using our previously developed approach, PATHOME [8], an in-depth, computational network systematic analysis, we successfully identified biomarkers for gastric and breast cancer tumor progression $[8,9]$. We also predicted GC progression to involve focal adhesion subpathways in, which rely on RHOA for cytoskeletal $[8,10]$. RHOA is frequently overexpressed in Japanese and Chinese GC patient tumors [11, 12], while GC datasets from The Cancer Genome Atlas (TCGA) depository [13] showed RHOA mutations, not mere overexpression, in diffuse type GC tumors $[14,15]$. Based on RHOA's involvement in other cancers, in this study, we herein investigated whether RHOA inhibitors could successfully be identified as an evidence-based, biomarker-driven therapeutic option for GC patients. In particular, we observed that $R H O A$ upregulation, concomitant with reduced $R H O B$ downregulation, was a common occurrence in Asian GC tumors. Moreover, RHOA perturbation resulted in strong inhibition of GC cell proliferation and tumor growth. Lastly, we developed an evidence- and hypothesis-driven, cheminformatics approach to successfully identify five candidate RHOA inhibitors. The latter represents a straightforward and innovative method for the development of promising, enzyme-binding small molecules for suppressing oncogenic signaling pathways

\section{RESULTS}

\section{Identification of $\mathrm{RHOA}$ upregulation in Asian gastric cancer}

In our previously study, we identified focal adhesion pathways as significant to GC by transcriptomic analysis using PATHOME [8]. Use of an independent Asian RNA-seq dataset [GEO accession: GSE36968 (24 GC, 6 normal samples) [16] validated our previous finding by showing RHOA association with actin cytoskeleton signaling, one of the top 31 pathway clusters (Figure 1A). In particular, we show here that chemokine signaling, focal adhesion, and other cancer-related (Cluster 6, 17, 20, 26 and 31) pathways (Figure 1A, right panel), all involve RHOA. Using the same dataset, we showed RHOA expression levels by tumor stage (Figure 1B; see sample information in Supplementary Table S1), revealing statistically significant (p-value 0.0409 by contrast in one-way ANOVA) association with Stage I tumors (see Supplementary Table S1), as compared to normal stomach (Figure 1B).

Using the TCGA GC dataset [13], we next compared RHOA-related gene expression patterns between 77 Asian and 172 Caucasian cases (Figure 2). In Asian GC, RHOA expression showed significant differences between disease stages (p-value 0.032 by ANOVA test) (Figure 2A; see sample information in Supplementary Table S1). Also, for Figure 2A, we performed another statistical test, 1,000 random samplings without replacement. In each random sampling, we permuted stage labels against the original data, subsequently calculating F-statistic. After 1,000 random samplings, we obtained the distribution of F-statistic. For example, if the observation of F-statistic for the original data as $f_{\text {obs. }}$, the empirical p-value was obtained by $\operatorname{Pr}\left(\mathrm{F}>f_{\text {obs }}\right)$. As a result, the empirical $\mathrm{p}$-values for Asian and Caucasian were 0.030 and 0.054 , respectively.

No significant differences were seen between the two groups with regard to the molecular subtypes characterized by TCGA (e.g., Epstein-Barr virus, microsatellite instability, genome stability, and chromosome instability) (Figure 2B). 2 of the 77 Asian GC tumors $(2.5 \%)$ showed RHOA mutations, as did 12 of the 172 Caucasian tumors (7.0\%) (Figure 2C, 2D). Due to the limited number of $R H O A$ mutations, the lack of significance should be carefully interpreted. Thus, from our comparison of the Asian vs. Caucasian datasets, we observed significant Asian GC RHOA upregulation, allowing us to proceed further to identify key genes in the RHOA-associated actin cytoskeleton signaling pathway. 


\section{GC cell lines shows RHOA expression level- dependent growth inhibition upon RNAi gene knockdown}

In addition to our tumor studies described above, we examined RHOA signaling, as a potential therapeutic target, in living cells. For this purpose, RHOA protein expression was assessed in $25 \mathrm{GC}$ cell lines. As shown in Figure 3A and 3B, high-to-medium
RHOA expression levels were observed in a majority of GC cells examined. Those results are consistent with our immunohistochemistry (IHC) studies (Figure 3B). IHC RHOA expression levels were ranked as follows: $2.5-3.0$ as high-, $2.0-2.5$ as medium-, and grades $\leq 2.0$ as low-expression. Thus, SNU-484 and SNU-601 GC cells were classified as high-expressing, NCC-19 as mediumexpressing, and AGS, NCI-N87, MKN45, and SNU-1967 as low-expressing cell lines (Figure $3 \mathrm{~A}$ and $3 \mathrm{~B}$ ).

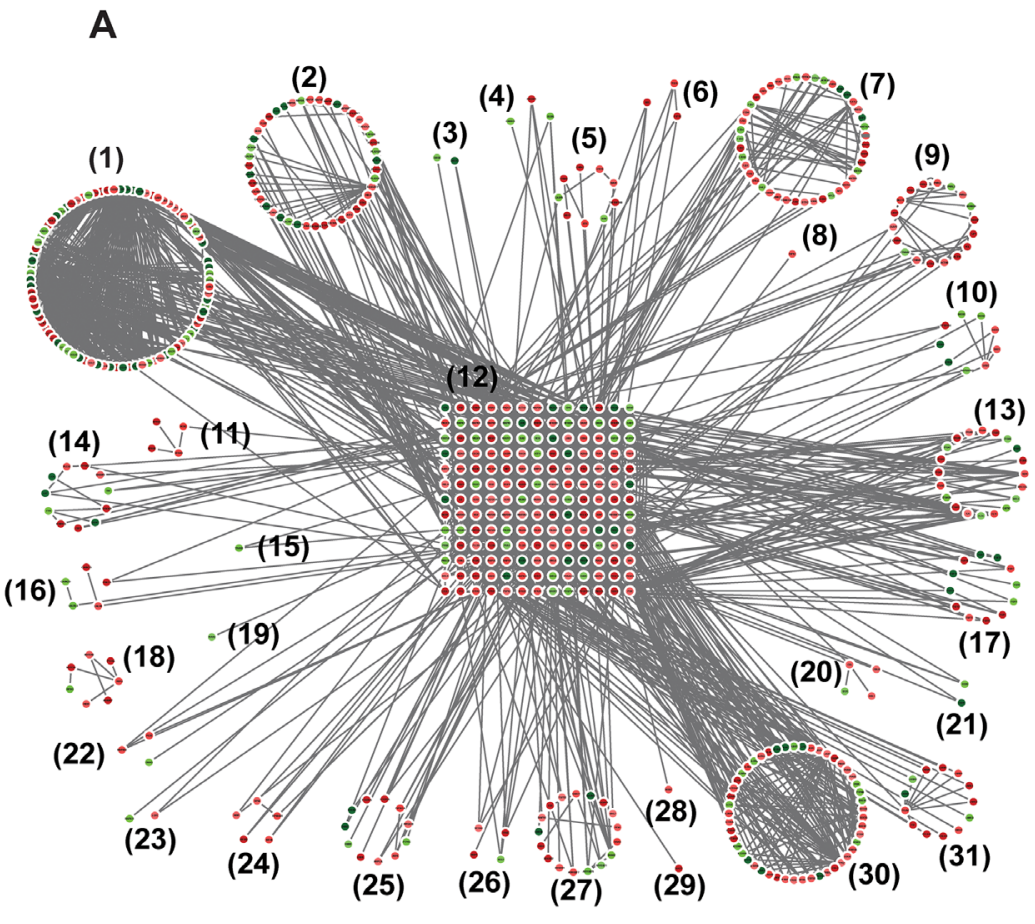

\begin{tabular}{crl}
\hline Cluster & KEGG_ID & \\
\hline 1 & hsa04062 & Chemokine signaling pathway \\
2 & hsa04010 & MAPK signaling pathway \\
3 & hsa04062 & Chemokine signaling pathway \\
4 & hsa04662 & B cell receptor signaling pathway \\
5 & hsa04115 & p53 signaling pathway \\
6 & hsa05215 & Prostate cancer \\
7 & hsa04310 & Wnt signaling pathway \\
8 & hsa04623 & Cytosolic DNA-sensing pathway \\
9 & hsa04520 & Adherens junction \\
10 & hsa04722 & Neurotrophin signaling pathway \\
11 & hsa04360 & Axon guidance \\
12 & Cross-talk & Cross-talk genes among other KEGG pathways \\
13 & hsa04510 & Focal adhesion \\
14 & hsa04650 & Natural killer cell mediated cytotoxicity \\
15 & hsa04920 & Adipocytokine signaling pathway \\
16 & hsa04916 & Melanogenesis \\
17 & hsa05218 & Melanoma \\
18 & hsa04330 & Notch signaling pathway \\
19 & hsa05142 & Chagas disease \\
20 & hsa05220 & Chronic myeloid leukemia \\
21 & hsa04660 & T cell receptor signaling pathway \\
22 & hsa04912 & GnRH signaling pathway \\
23 & hsa04664 & Fc epsilon RI signaling pathway \\
24 & hsa04910 & Insulin signaling pathway \\
25 & hsa04666 & Fc gamma R-mediated phagocytosis \\
26 & hsa05200 & Pathways in cancer \\
27 & hsa04810 & Regulation of actin cytoskeleton \\
28 & hsa05131 & Shigellosis \\
29 & hsa04621 & NOD-like receptor signaling pathway \\
30 & hsa04630 & Jak-STAT signaling pathway \\
31 & hsa05222 & Small cell lung cancer \\
\hline & & \\
& & \\
\hline
\end{tabular}

B

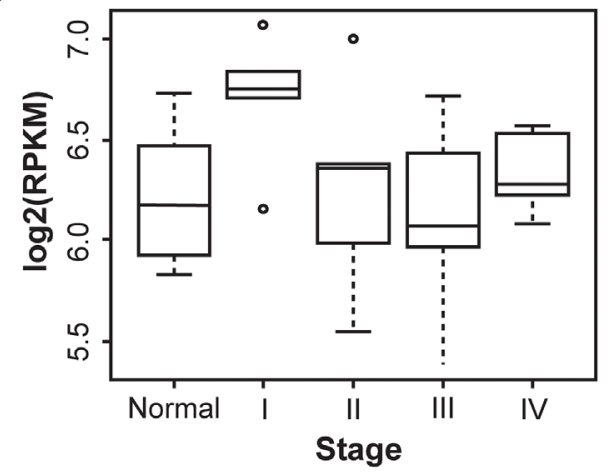

\begin{tabular}{cc} 
Hypothesis & p-value \\
\hline Normal vs. (Stages I \& II) vs. (Stages III \& IV) & $0.242^{\mathrm{a}}$ \\
Normal vs. Stage I vs. Stage II vs. Stage III vs. Stage IV & $0.129^{\mathrm{a}}$ \\
Normal vs. Stage I & $0.0409^{\mathrm{b}}$ \\
Normal vs. (Stages I \& II) & $0.1858^{\mathrm{b}}$ \\
Normal vs. (Stages III \& IV) & $0.9365^{\mathrm{b}}$ \\
(Stages I \& II) vs. (Stages III \& IV) & $0.1233^{\mathrm{b}}$ \\
Stage I vs. (Stages II \& III \& IV) & $0.0207^{b}$ \\
Stage I vs. (Normal \& Stages II \& III \& IV) & $0.0166^{b}$ \\
Normal vs. (Stages I \& II \& III \& IV) & $0.4258^{\mathrm{b}}$ \\
\hline
\end{tabular}

a One-way ANOVA test

${ }^{b}$ Contrast test in ANOVA

Figure 1: Network analysis in a Korean GC RNA-Seq dataset shows an underlying GC tumor oncogenetic network, under various signaling contexts. A. PATHOME analysis of Korean GC dataset GSE36968 resulted in 31 functional clusters consisting of significant KEGG subpathways. The clusters were assigned to their corresponding KEGG pathway titles. The network diagram showed upregulated genes in red and downregulated genes in green (left panel), and the designated KEGG pathway titles noted in the right table. The network contained RHOA as a "cross-junction" involved in several pathways (see details in the main text). Pathways related to RHOA are marked red. B. From previous Asian GC samples (deposited in GEO; GSE36968), RHOA expression was inspected throughout $\mathrm{GC}$ tumor stages. The $\mathrm{x}$-axis represents stage, and the $\mathrm{y}$-axis $\log _{2}$-scaled RPKM. Stage I patients showed higher RHOA gene expression compared to other stage patients, including normal controls. 
A

Asian

Caucasian
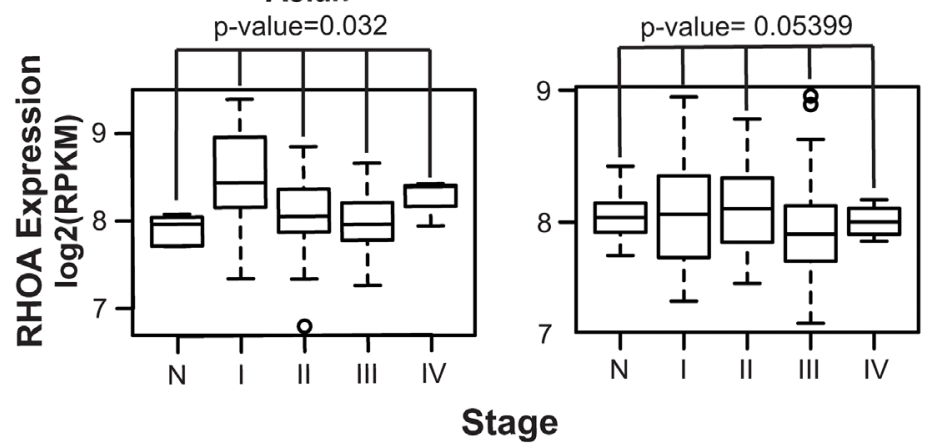

B

n.s.

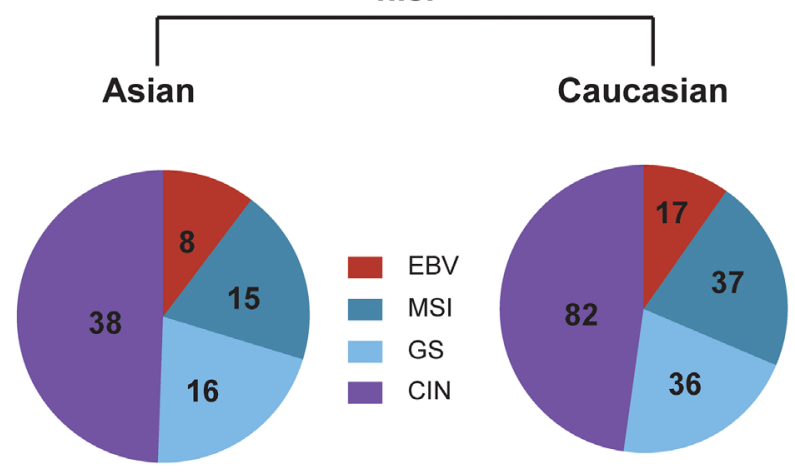

C

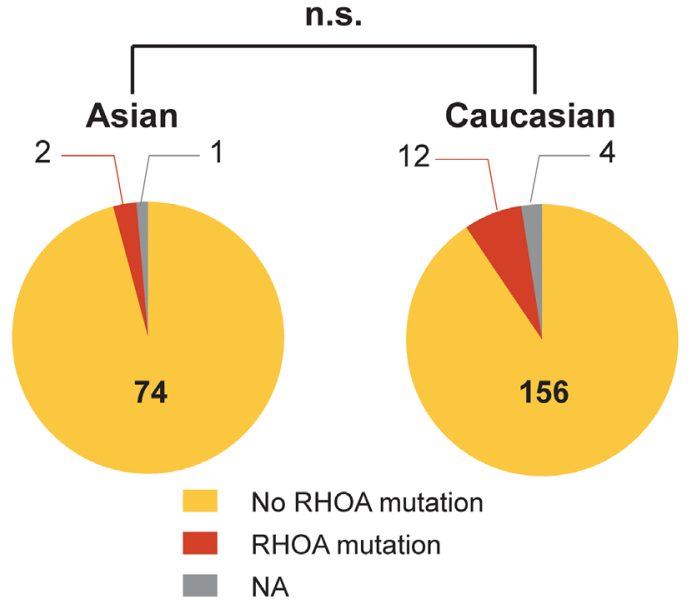

D

\section{Asians with RHOA mutations}

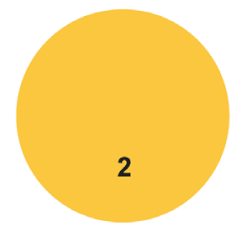

\begin{tabular}{lll}
\cline { 1 - 1 } Lauren Class & & $\#$ \\
Diffuse & & 2 \\
Intestinal & & 0 \\
Mixed & & 0 \\
NA & 0
\end{tabular}

Caucasians with RHOA mutations

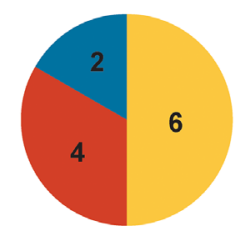

\begin{tabular}{lll}
\cline { 1 - 1 } Dauren Class & & $\#$ \\
Intestinal & & 4 \\
Mixed & & 2 \\
NA & & 0
\end{tabular}

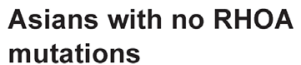
mutations

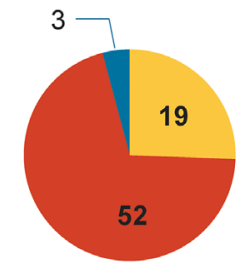

\begin{tabular}{lll}
\cline { 1 - 1 } Lauren Class & & $\#$ \\
Diffuse & & 19 \\
Intestinal & & 52 \\
Mixed & & 3 \\
NA & & 0
\end{tabular}

\section{Caucasians with no RHOA mutations}

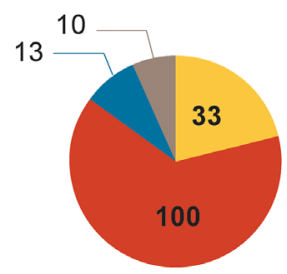

\begin{tabular}{lll}
\cline { 1 - 1 } Lauren Class & & $\#$ \\
Intestinal & & 33 \\
Mixed & & 130 \\
NA & & 10
\end{tabular}

Figure 2: $\mathbf{R H O A}$ expression analysis shows difference in Asian vs. Caucasian. A. $R H O A$ mRNA expression levels, by cancer stage in both Asian and Caucasian races, showed RHOA expression to significantly associate (p-value 0.032 by one-way ANOVA) with Asian GC disease stages, but not in Caucasians. In particular, RHOA up-regulation in Stage I, compared to normal, is shown. B. molecular subtypes between TCGA Asian and Caucasian GC patients. By using cBioPortal (data version: Stomach Adenocarcinoma (TCGA, Nature 2014)), the proportions between the two races, in terms of molecular subtypes, were not statistically different. C. RHOA mutation between TCGA Asian and Caucasian datasets. By using cBioPortal (as above), the proportions between the two racial groups, in terms of RHOA mutations, were not statistically different. D. RHOA mutations, as compared between TCGAAsian and Caucasian data, according to Lauren class. Diffuse type was bolded to show enrichment of mutation compared to intestinal and mixed. As shown, the proportions between the two ethnicities in terms of RHOA mutations and Lauren class were not statistically different. 
A
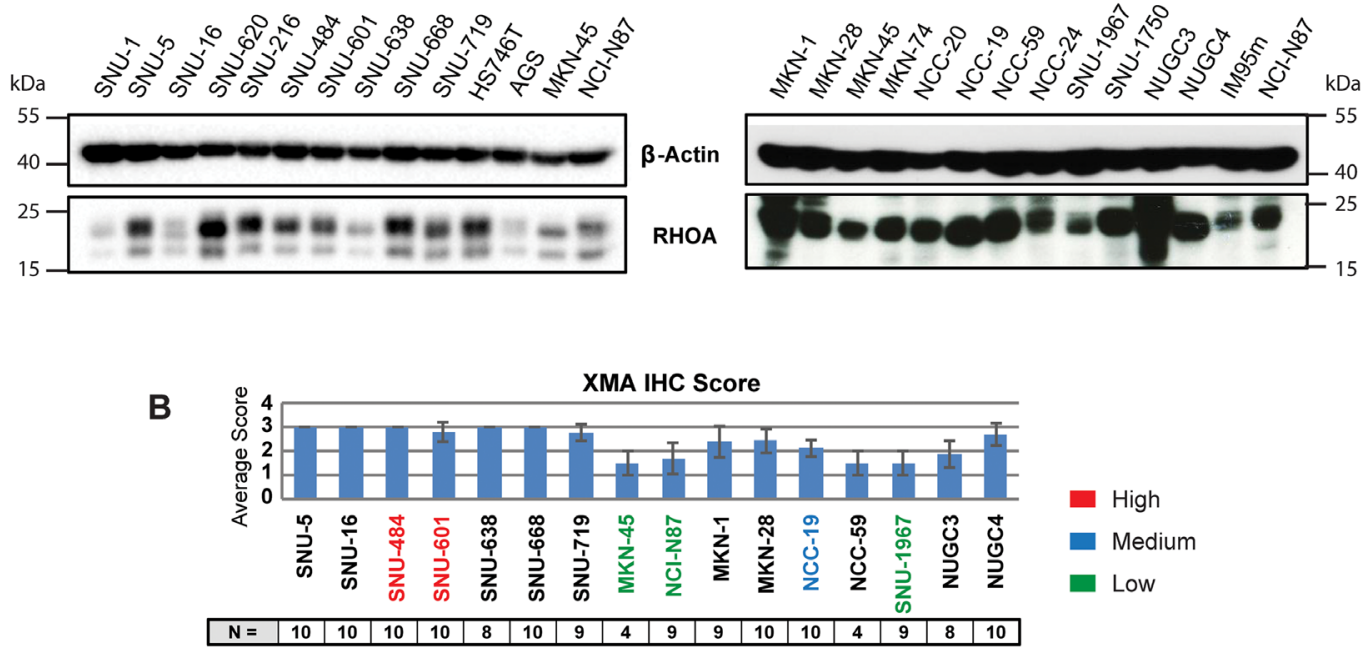

C
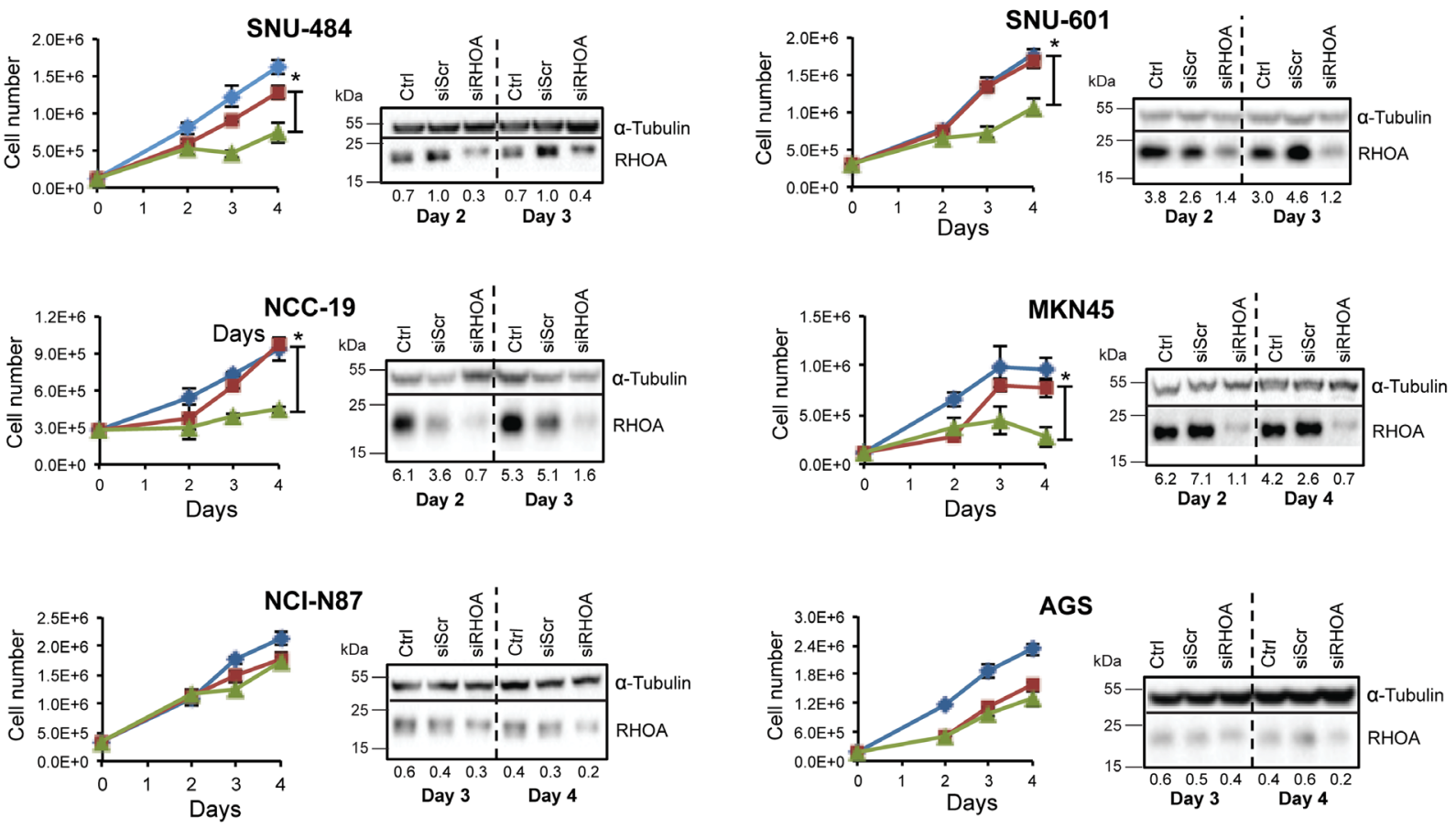

AGS
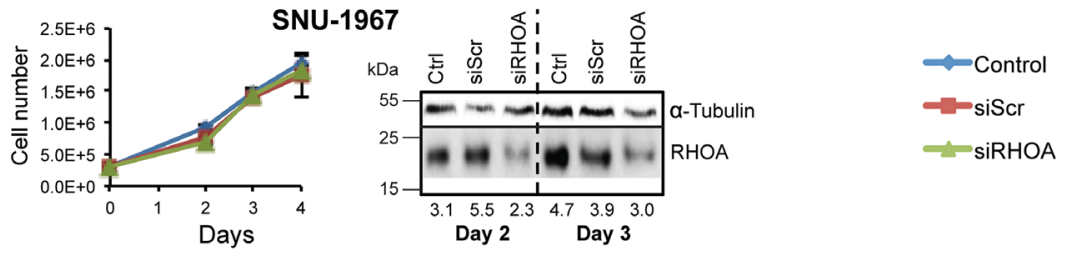

Figure 3: Gene knockdown of $\mathrm{RHOA}$ in GC cell lines show differences in cell proliferation. RHOA protein expression evaluation on GC cell line panel shows various levels of protein expression. A. Western blot analysis of RHOA expression on a 26$\mathrm{GC}$ cell line panel. B. Immunohistochemistry against RHOA of tissue microarray of cell lines that were successfully established into xenograft models graded from $0-3$. (2.5 3: high RHOA-expressing cells-red; 2 2.5: medium RHOA-expressing cells-blue; $<2$ : low RHOA expressing cells-green). $\mathrm{N}$ is the number of repeats per cell line. C. cell proliferation results after siRHOA gene knockdown. Harvested cells were mixed with Trypan blue (1:1) and live cells were counted. High RHOA-expressing GC cell lines, such as SNU-484 and SNU-601, showed decreased cell proliferation, whereas the RHOA-low expressing cell line SNU-1967 showed little effect (asterisks $(*)$ indicate p-values $<0.05$ ). Western blot analysis showed a decrease in RHOA protein after siRHOA infection of numerous GC cell lines. 
To further study RHOA function in GC cells, we performed siRNA knockdown and observed cell viability in the 7 cell lines mentioned above. Growth inhibition directly correlated with the level of RHOA knockdown. For example, poorly knocked down GC cell lines (e.g., AGS, NCI-N87 and SNU-1967), as confirmed by Western blot analysis, showed less growth inhibition than those with strong RHOA knocked down (e.g., SNU-484, SNU601, MKN45 and NCC-19 cells) (Figure 3C). Except for MKN45 cells, the high RHOA-expressing cell lines showed greater $\mathrm{siRHOA}$ knockdown, and were not viable, while the low-expressing cell lines were less sensitive to siRHOA knockdown. AGS cells however, were sensitive to the transfection reagent (data not shown), thus precluding their further analysis.

We also examined cell cycle distribution, showing that most siRHOA treated cells exhibited increased apoptosis (i.e., sub- $\mathrm{G}_{0}$ cellular debris), in positive correlation with RHOA knockdown (Figure 4A and Supplementary Figure S1). Although siRHOA knockdown minimally inhibited growth of some RHOA lowexpressing cells, it might possibly hinder actin-related cell functions such as migration. To assess the effect of $R H O A$ knockdown on the migration phenotype, we performed "wound healing" assays on the three (AGS, NCI-87 and SNU-1967) low RHOA-expressing cell lines that had reduced proliferation upon further RHOA knockdown. Loss of migration was not observed in any of the three cell lines when comparing siScr vs. siRHOA treatment (Figure 4B). Interestingly, RHOA knockdown increased migration in SNU-1967 cells to $63.3 \%$ wound closure, compared to $34.7 \%$ closure for siScr-transfected cells. These results show that cell lines with differential endogenous RHOA expression differ in their responses to RHOA knockdown. Namely, cell growth was more inhibited in cell lines with higher RHOA expression, than in cell lines with low expression, with the latter also demonstrating impaired migration, upon $R H O A$ knockdown.

\section{shRNA knockdown of $R H O A$ inhibits tumor growth in GC xenograft models}

To observe RHOA knockdown effect in vivo, SNU484 and SNU-601 cells were selected to generate stable cell lines having shRNA-knocked down RHOA, for subsequent mouse xenograft studies. Figure $5 \mathrm{~A}$ shows, by Western blot, different knockdown levels of distinct clones shown by Western blot. A mixture of the greatest RHOAknock-down (lowest RHOA expressing) cells (clones \#4 and \#5 for SNU-484 and \#2 and \#4 for SNU-601) was engrafted to two groups of nude mice with control- or RHOA shRNA-transfected SNU-484 and SNU-601 cells. Tumors from RHOA knockdown cells grew just above the baseline, showing significant differences in size, as compared to the control tumors ( $p$-value $<0.05$; Figures 5B and 5C). In SNU-601 xenografts, shRNA-
RHOA tumor growth was completely inhibited. Overall, of the two GC xenograft models, RHOA knockdown suppressed tumor growth in both, reaffirming its role in $\mathrm{GC}$ oncogenesis.

\section{RHOA RNAi knockdown shows RHOA-RHOB potential crosstalk}

We next explored the subpathway(s) involved in GC cell growth inhibition upon siRHOA transfection, by assessing gene expression levels were evaluated by RT-PCR. Two (SNU-484 and SNU-601) RHOA highexpressing cell lines and one (SNU-1967) low-expressing cell line (Figure 6A) we used. In all three cell lines, the RHOA upstream genes, ARHGEF11, ARHGEF12 and $A R H G A P 26$ were upregulated (Figure 6B), as was a downstream gene, $P L D 1$.

Interestingly, $R H O B$, a Rho family gene and homolog of RHOA, was consistently upregulated in all three cell lines (Figure 6B). Overall, the expression levels of RHOA pathway genes were upregulated in SNU-1967 ( $\geq 2$-fold), compared to SNU-484 and SNU601 cells (Figure 6B). In the GC dataset as mentioned above, $R H O B$ was significantly downregulated in tumor samples compared to normal tissues in Asian GC, but not in Caucasian GC tumors (Figure 6C). Conversely, the phospholipases gene D1 (PLD1) was upregulated in Asian, but not in Caucasian GC samples. These findings also showed that the expression levels of several $R H O A$ related genes were altered upon mRNA perturbation.

We also performed another statistical measurement (for Figure 6C) by using 1,000 random samplings without replacement, for $R H O B$ and $P L D 1$, as described above. For $R H O B$, the empirical p-values for Asian and Caucasian were 0.000 and 0.361 , respectively. And, for $P L D 1$, the empirical p-values for Asian and Caucasian were 0.009 and 0.077 , respectively. These empirical p-values of PLD1 and $R H O B$ agreed with the $\mathrm{p}$-values results of ANOVA tests.

\section{In Silico screening of small molecules specifically binding to RHOA}

Our overall procedure for our virtual screening of target RHOA inhibitors is shown in Figure 7A. First, we searched PUBCHEM (pubchem.ncbi.nlm.nih.gov) [17] for compounds structurally similar to the known RHOA inhibitor, Rhosin [18], to measurement similarity (Tanimoto score). We further selected all compounds dockable to the mapped binding regions in the RHOA crystal structure. The potential binding regions defined as "clusters," for the dockable compounds, are shown in the right panel in Figure 7A. Finally, we applied binding specificity as well as drug-like physicochemical properties (Lipinski's rule of five) [19] for further filtering (see Materials and Methods). 
A

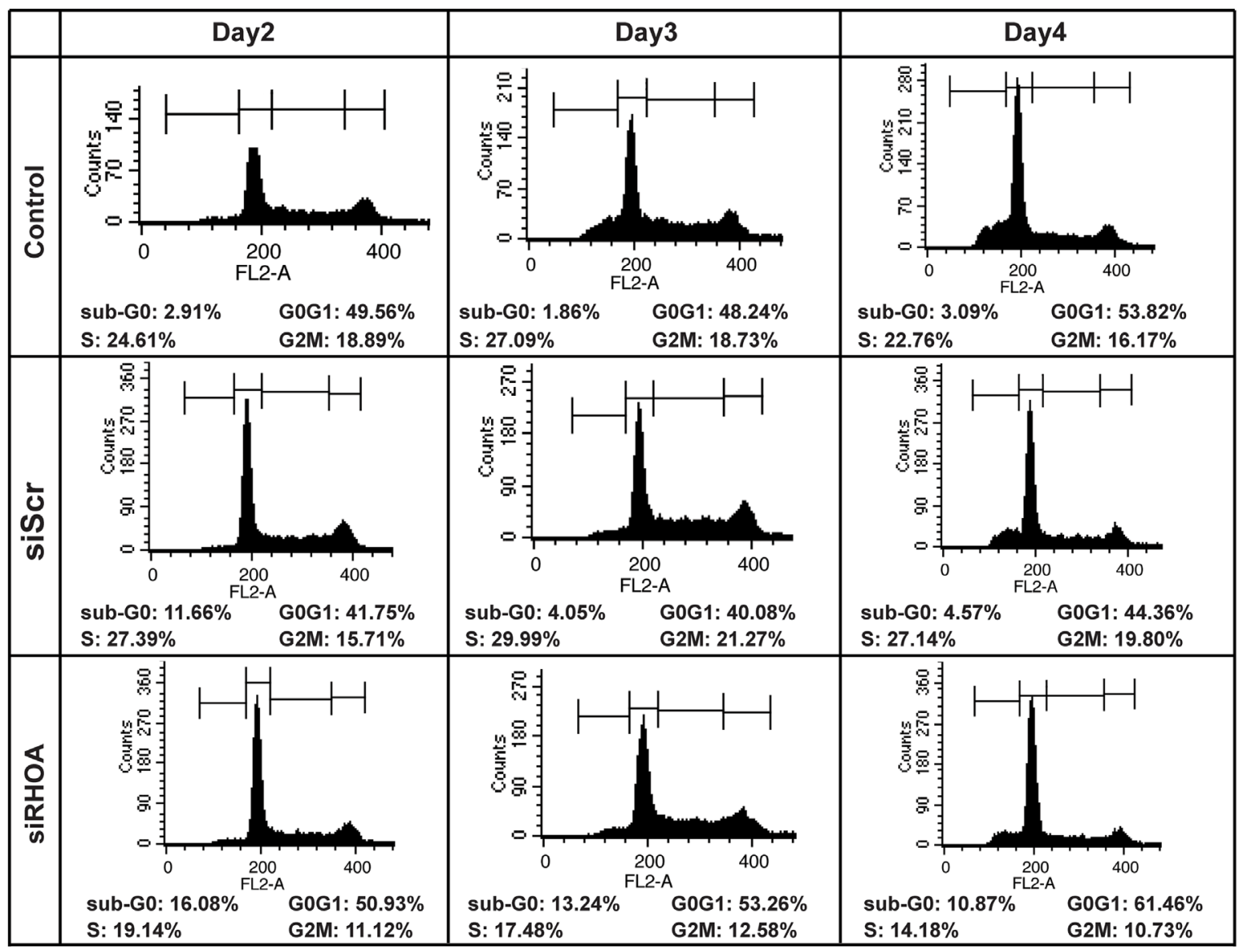

B

AGS

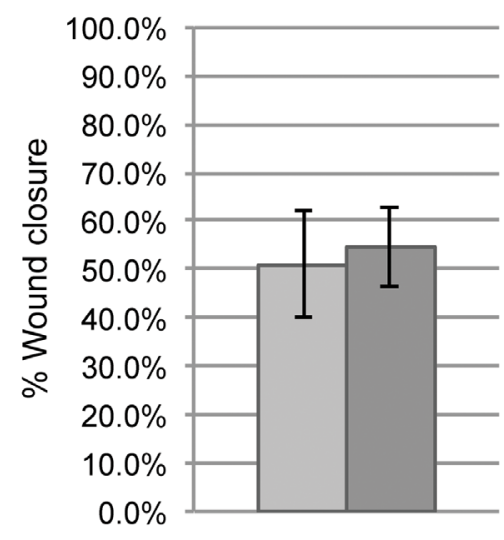

$\mathrm{NCl}-\mathrm{N} 87$

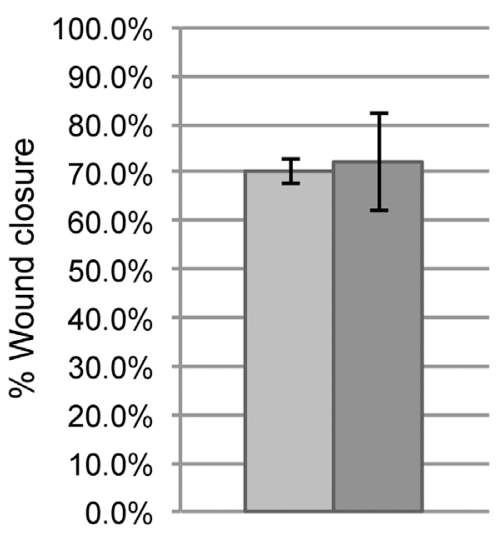

SNU-1967

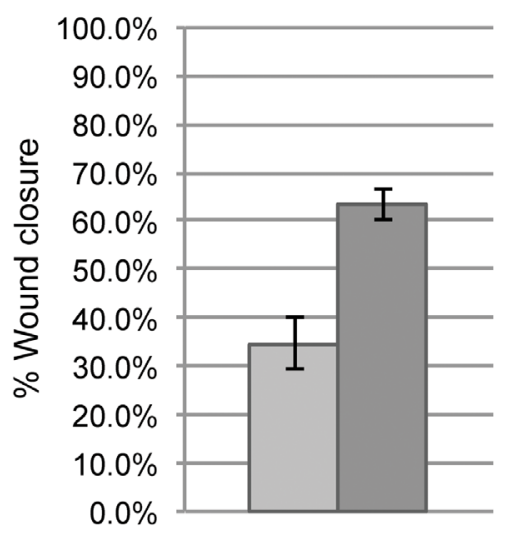

口siScr

$\square$ siRHOA

Figure 4: Gene knockdown of $\mathrm{RHOA}$ in GC cell lines show differences in cell function depending on RHOA protein expression. A. cell flow cytometry results showed that sub-G0 population (apoptotic) increased as $R H O A$ was knocked down upon $R H O A$ knockdown in SNU-601 cell line. B. Migration assay was performed on three low RHOA-expressing GC cell lines (AGS, NCI-N87 and SNU-1967) after RHOA knockdown by siRNA. AGS and NCI-N87 show little difference in migration between siScr and siRHOA, and SNU-1967 show increased migration upon RHOA knockdown. 
Of the 45 million compounds in PUBCHEM, we identified 41 with high $R H O A$-binding specificity (Figure 7A) based on the energy differences between the first and second binding energies of the compound (Figure 7B; details in Supplementary Methods). Due to the small volume of some of the compounds compared to the potential binding pocket volume, non-specific binding to multiple regions may occur even within the pocket. Consequently, the binding energy differences between these regions within the pocket were calculated to identify compounds with the greatest energy differences. These were then selected as specifically binding to a certain region, corresponding to the first and the second lowest-binding energies, referring to the effective binding specificity to the top region within any specific cluster.

From the 41 compounds, 7 representatives were chosen and tabulated (Figure 7C). Since the protein ARHGEF12 (LARG) physically binds to RHOA at the protein-protein interaction interface [20], we focused on this region to identify two compounds (PUBCHEM IDs 4654934 and 3785359 ) that bound to clusters 4 and 3 , respectively (Figure 7D). These two compounds could, computationally, directly bind to the protein-protein interaction interface of the LARG-RHOA complex. Another five compounds (PUBCHEM IDs 66954415, $58882795,52207176,26299905$ and 24535629, binding to

A

SNU-484

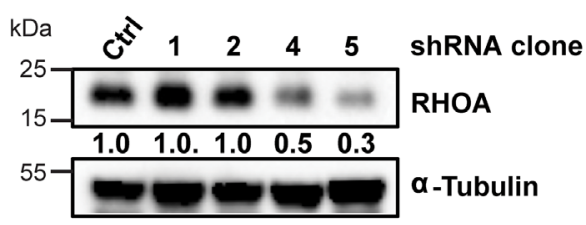

SNU-601

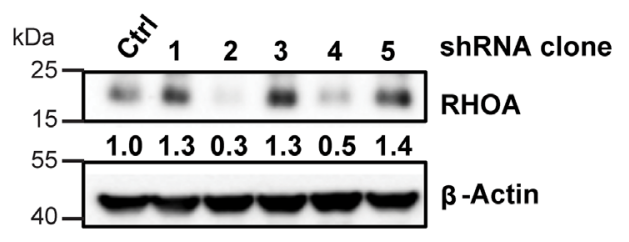

B

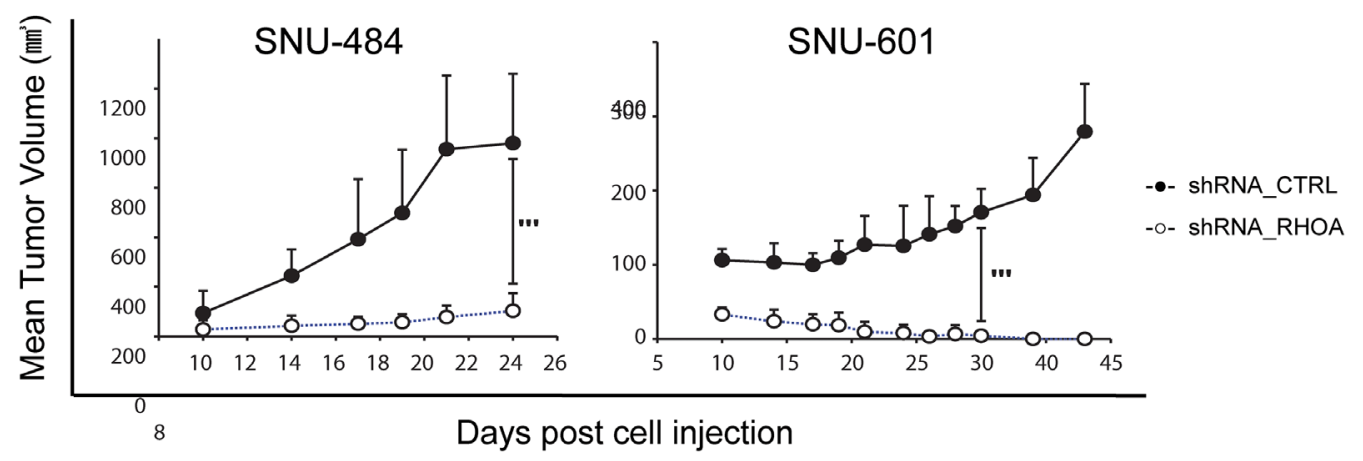

C

SNU-484

ShRNA_CTRL

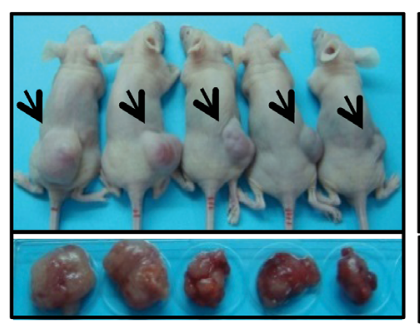

ShRNA_RHOA

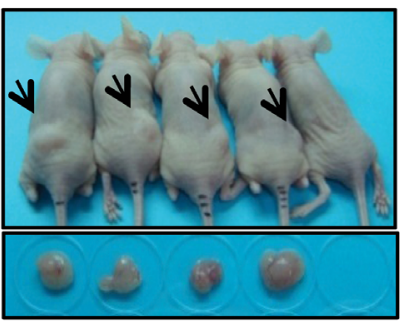

SNU-601 ShRNA_CTRL ShRNA_RHOA

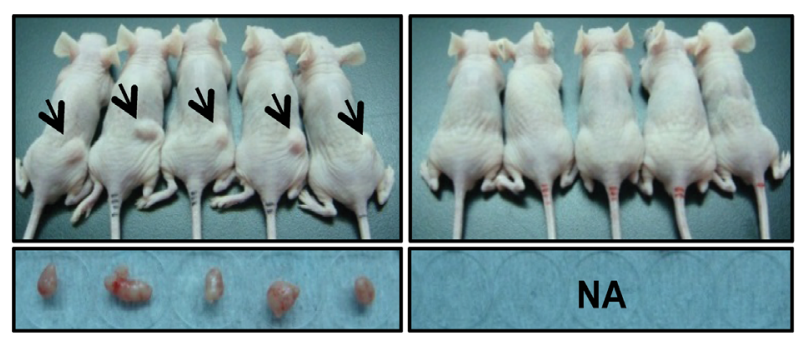

Figure 5: In vivo xenograft model of shRHOA shows decreased tumor size. A. Western blot analysis shows different knockdown efficiencies of each clone after lentiviral infection of RHOA shRNA. B. tumor growth was measured from day 10 of inoculation and was monitored until the animals were sacrificed. Tumor growth suppression was observed in RHOA shRNA knockdown tumors (SNU-484 and SNU-601), as compared to the various control groups. $* * *$-value: SNU-484p-value $=0.0061$, SNU-601 p-value $=0.0004)$ C. photograph of mice bearing xenograft tumors. Arrows indicate the location of the tumors. Tumor sizes from the RHOA knockdown cells were smaller in SNU-484 GC cells, and failed to grow in SNU-601 cells. 
A

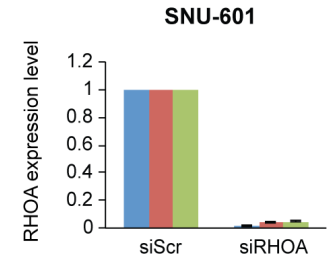

SNU-484

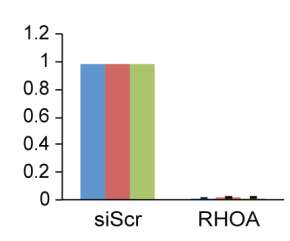

SNU-1967

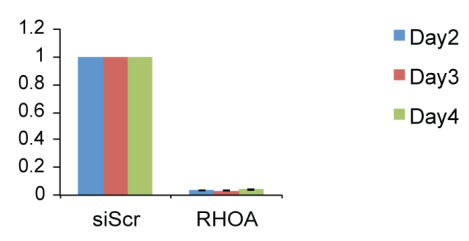

B
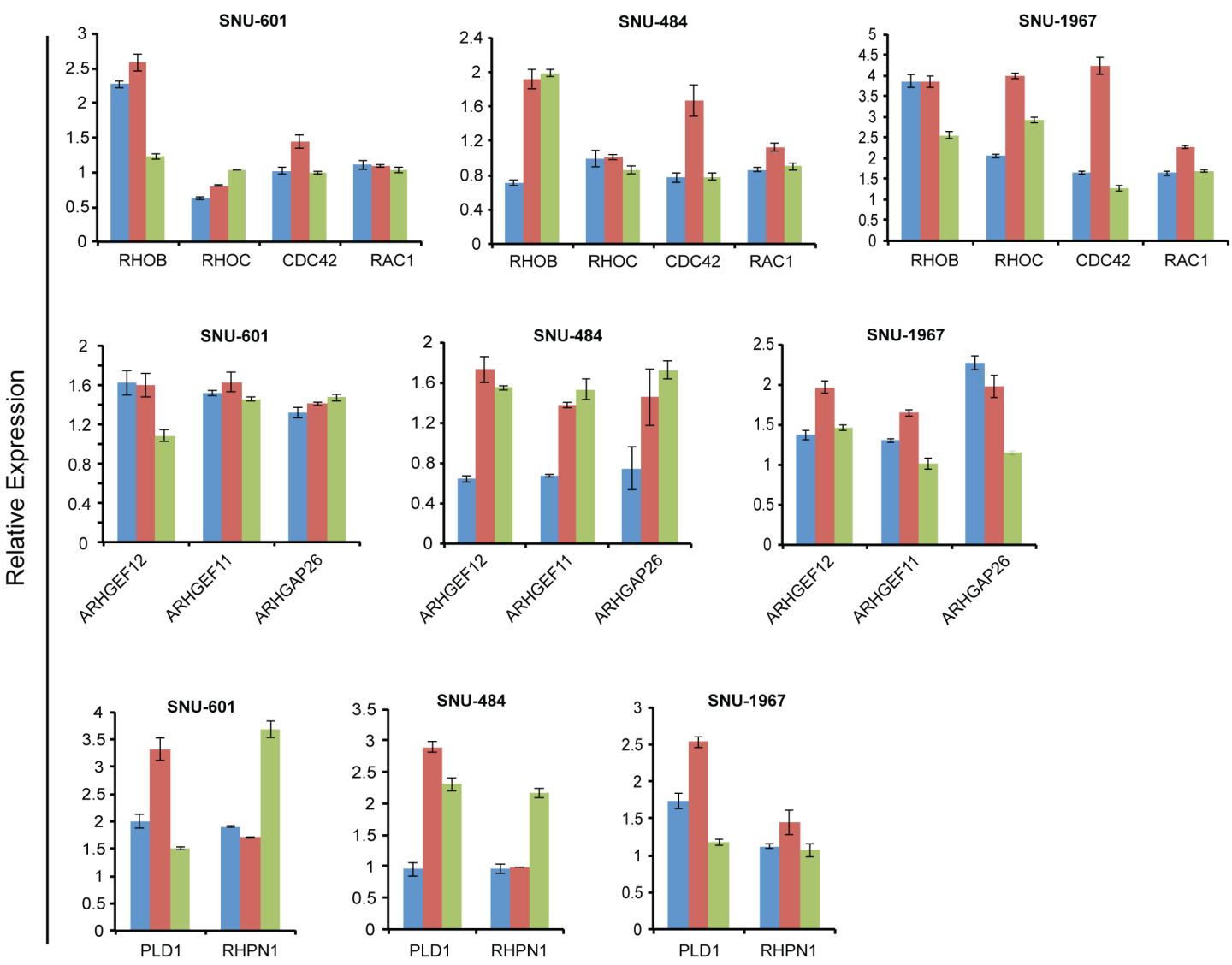

C
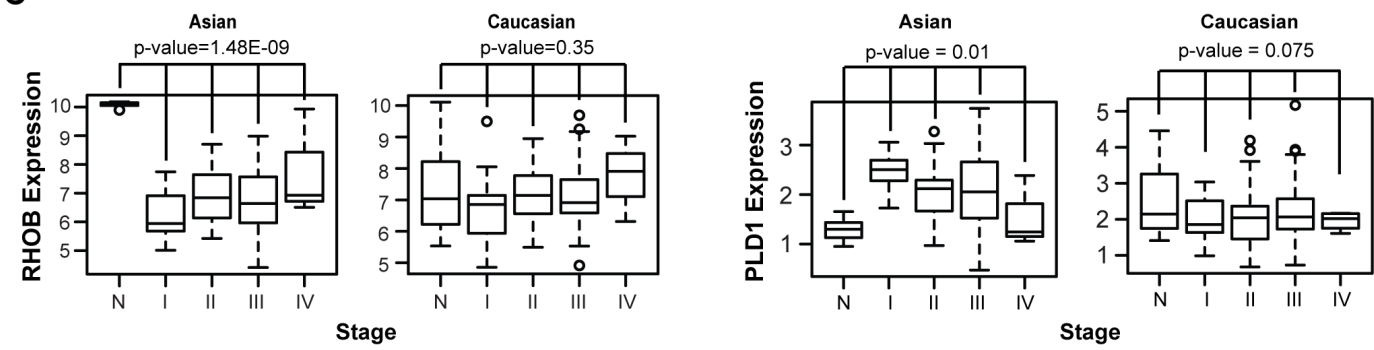

Figure 6: $R H O A$ knockdown results show unique patterns of downstream genes and induction of cell death. A. quantitative RT-PCR results of SNU-601 cells after RHOA siRNA knockdown. RHOA was successfully knocked down in RHOA high-expressing (SNU-484 and SNU-601) as well as low-expressing (SNU-1967) cell lines. B. mRNA expression level of various RHOA network genes were evaluated by RT-PCR after siRNA knockdown of RHOA. RHOB was consistently upregulated in 3 cell lines (SNU-601, SNU-484 and SNU-1967), but not other Rho family genes RHOC, CDC42 and RAC1. Upstream genes ARHGAP26, ARHGEF11 and ARHGEF12 were found upregulated in all three cell lines, as well as $P L D 1$, and to a lesser degree, RHPN1. C. RHOB is significantly downregulated in Asian, but not in Caucasian, GC (p-values by one-way ANOVA), and PLD1 shows upregulation in Asian GC. Gene expression was inspected with reference to normal samples and tumor stages. 
A

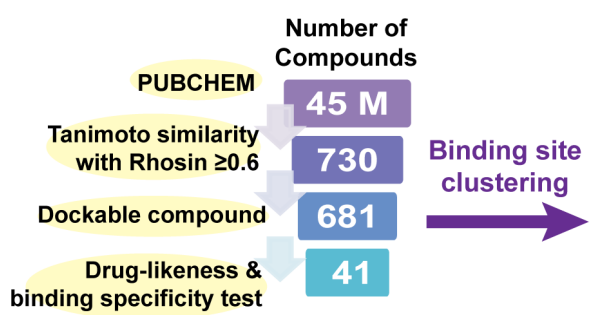

B

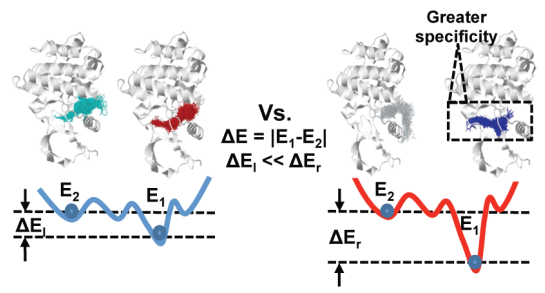

D
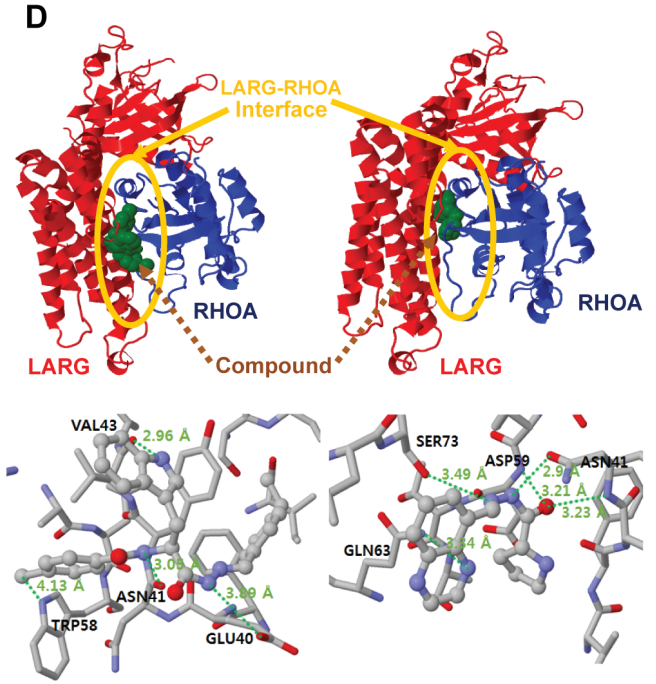

1) 5

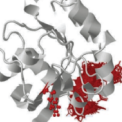

5)

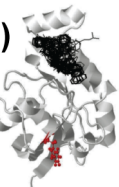

9)

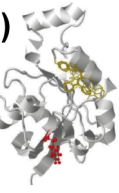

2)

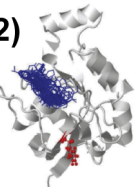

6)

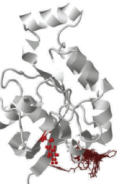

10)

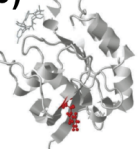

3)

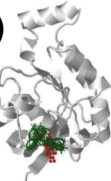

7)

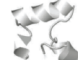

sont

30

11)

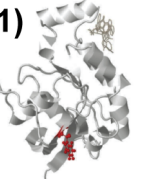

4)

3

$5 x^{2}$

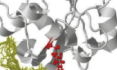

8)

85

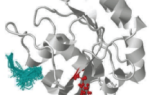

12)

2)

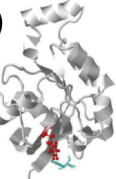

C

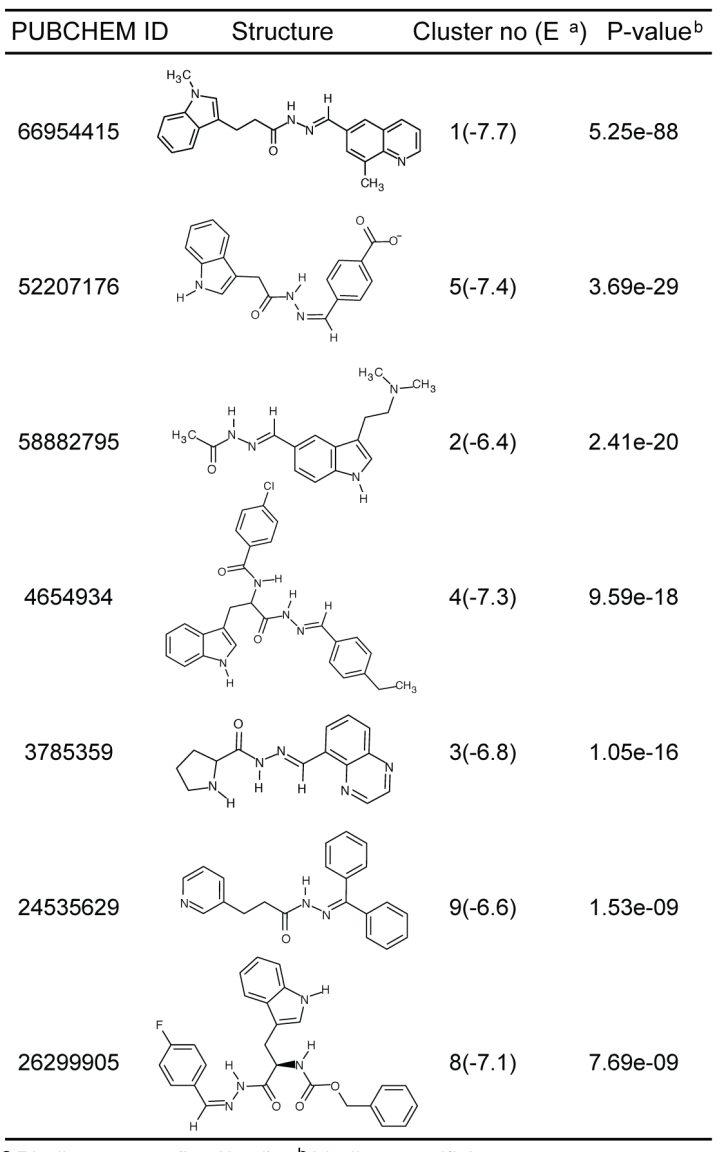

${ }^{a}$ Binding energy ( $\left.\mathrm{kcal} / \mathrm{mol}\right) ;{ }^{\mathrm{b}}$ binding specificity test

Figure 7: A systematic search of RHOA inhibitor small molecules suggests several candidates. A. number of compounds remaining after each round of filtering (left panel). The selected compounds were docked in different clusters of novel RHOA binding pockets (right panel). B. schematic binding-specific test figure. Smaller (left), and larger specific situations (right). $\mathrm{E}_{1}$ and $\mathrm{E}_{2}$ are the first and the second representative energies in binding clusters. Larger energy differences $\left(\Delta \mathrm{E}_{\mathrm{r}}\right)$ represent more specific compounds. $\mathrm{C}$. chosen RHOA candidate drug list tabulated by PUBCHEM ID, structural figure, cluster number and binding energy in parenthesis, and P-value calculated by binding-specific test. D. structure figures in cluster no. 4 (left) and no. 3 (right). (Upper panel) ARHGEF12 (LARG) and RHOA are drawn by red and blue cartoon images, respectively. The LARG-RHOA interfaces are denoted by the yellow circles. The bound compounds of clusters no. 4 and no. 3 are drawn by green spheres (lower panel). Hydrogen bonds, depicted by thick dotted lines with the distance between donor and acceptor atoms. Interacting residues with the compound are expressed by residue names and respective numbers. 
cluster numbers 1, 2, 5, 8 and 9, respectively) were found on other surfaces (i.e. not in the LARG-RHOA interface). Thus, these two inhibitors identified above may have an allosteric effect, indirectly preventing LARG-RHOA interaction by altering the RHOA conformation.

\section{Synthesis and biological evaluation of potential inhibitors targeting RHOA}

Considering our computational and laboratory results, we focused on rigorous design of hydrazide homologues, a functional group found in several of the successfully identified compounds. Based on the results from our in silico screening, we designed a hydrazide functional group serving as a spacer skeleton, with the structural variation of the R moiety shown in Figure 8A. For greater diversity, we rationalized that structurally similar compounds would likely exhibit similar biological activity, and we thus fixed the piperonyl group and hydrazide spacer moiety in the compound structure. By varying the $\mathrm{R}$ moiety for structural modification, we expected different biological activities, depending on the moiety's coverage. For the purpose of achieving wide diversity by navigating through the relevant chemical space, five distinct compounds, representing different chemical properties, were designed and synthesized. Of these, the compound JK-122 was synthesized to evaluate the activity of a non-polar phenyl group series as an $\mathrm{R}$ moiety. Compared to JK-122, JK-121 was synthesized to evaluate the effect of a sulfonyl functional group series in the hydrazide spacer. JK-123 and JK-124 were synthesized to assess the necessity and the activity of a hydrophilic functional group series, such as the nitrogen or the hydroxyl group, to participate in hydrogen bonding. JK125 , by contrast, was synthesized for assessing the effect of replacing an aromatic group with an aliphatic group. The synthetic strategy by piperonal treatment of selected hydrazides for the preparation of compounds JK-121 125, is depicted in Figure 8A.

Protein-small molecule interactions were further analyzed by surface plasmon resonance (SPR) to determine the binding affinity as well as the on/off-rate of each compound to RHOA protein. Of the five compounds tested, JK-122 showed the lowest dissociation constant, $\mathrm{K}_{\mathrm{D}}$ at $6.9 \pm 0.4 \mu \mathrm{M}$ (Figure $8 \mathrm{~A}$ ) and $\mathrm{k}_{\mathrm{d}}$ (dissociation rate or 'off rate') of $1.4 \mathrm{e}-3 \pm 0.0001 \mathrm{~s}^{-1}$, both acceptable values for protein binding of small molecule. Thus, of the five compounds we tested, the kinetics data showed JK-122 to be the best for further investigation as a RHOA inhibitor.

For in vitro compound assessments, $7 \mathrm{GC}$ cell lines (selected based on high, mid or low-RHOA expression levels) were treated with $20 \mu \mathrm{M}$ of JK-121 125. JK-122 showed up to $50 \%$ growth inhibition at $20 \mu \mathrm{M}$, while the others had little to no effect (Figure 8B). A previous Rhosin identification study reported that an aromatic ring on both ends of the molecule may be necessary, and our results confirm this [18]. Thus, our synthetic strategy and these "lead" compounds may provide valuable information for further optimization of novel RHOA inhibitors.

\section{DISCUSSION}

In this study, we systematically assessed the role of RHOA pathway dysregulation in GC, and the feasibility of clinically targeting RHOA for GC therapy. Our results suggest $R H O A$ as a genomic (mutation, amplification, etc.) and transcriptomic (overexpression) biomarker and therapeutic target in Asian GC patients. By showing RHOA upregulation as predominant in Korean GC tumors, and mutation more prevalent in Caucasian GCs, we demonstrate GC to have racial-specific etiologies [21]. Specifically, differences in the expression of $R H O A$ and its homolog, RHOB, implicate Rho GTPases in differential GC (Figure 6). Such findings will ultimately be important for precision medicine, as biomarker-driven cancer therapies inevitably rely on pathway differences for patient stratification. In the short term, however, such knowledge could be valuable for diagnostic purposes.

The finding of variable RHOA expression in diverse GC cell lines, is not surprising, considering the high degree of heterogeneity of this specific tumor type [21]. Proper cell line selection is critical for drug target preclinical studies, as drug efficacy may differ between cell lines (and tumor subpopulations), preventing missed "druggable" targets. For example, for preclinical evaluation of trastuzumab, NCI-N87 and OE19 were the only two HER2-overexpressing GC cell lines reported by Wainberg et al. [22]. Assessment of diverse cell lines could be highly valuable in clinical settings, such as patient stratification based on oncogenic signaling [23]. Here, we evaluated the downstream effects of RHOA knockdown in two RHOA high-expressing GC cell lines, SNU-484 and SNU-601, showing different expression patterns, compared to SNU-1967, a RHOA low-expressing cell line (Figure 6). This result implies that the RHOA signaling pathway may yield distinct phenotypes, depending on pathway gene expression levels or connection to other pathway networks (i.e. "crosstalk") [24]. Consequently, the detailed mechanisms of RHOA pathway activators and inhibitors need further investigation, with proper cell line selection for distinct druggable targets, relying upon diverse, physiologically relevant disease models for accurate results.

Upon $R H O A$ knockdown, the apoptotic gene $R H O B$ $[24,25]$, was consistently upregulated. It is possible that such anti-correlation occurs downstream of RHOA-RHOB, or depends on an independent subpathway. Thus, it is unclear which downstream genes are up-/down-regulated by RHOA knockdown, and further investigation of network genes is necessary to validate those subpathways. While crosstalk between these two genes remains unclear, decreased cell growth in knocked-down RHOA high-expressing cells 
A

\begin{tabular}{|c|c|c|c|c|c|}
\hline $\begin{array}{c}\text { Compound } \\
\text { No. }\end{array}$ & $\mathrm{R}$ & Product Structure & $k_{a}\left(M^{-1} s^{-1}\right)$ & $k_{d}\left(s^{-1}\right)$ & $\mathrm{K}_{\mathrm{D}}$ \\
\hline JK-121 & & & $45 \pm 2$ & $3 e-3 \pm 0.0001$ & $67 \pm 3 \mu \mathrm{M}$ \\
\hline
\end{tabular}

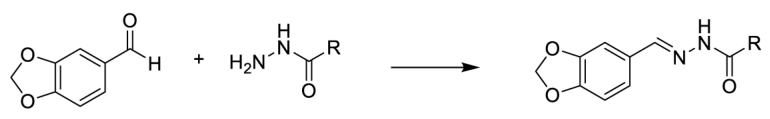

\begin{tabular}{|c|c|c|c|c|c|}
\hline $\begin{array}{l}\text { Compound } \\
\text { No. }\end{array}$ & $\mathrm{R}$ & Product Structure & $k_{a}\left(M^{-1} s^{-1}\right)$ & $k_{d}\left(s^{-1}\right)$ & $K_{D}$ \\
\hline JK-122 & & & $200 \pm 1$ & $1.4 \mathrm{e}-3 \pm 0.0001$ & $6.9 \pm 0.4 \mu \mathrm{M}$ \\
\hline JK-123 & & & $99 \pm 3$ & $1.6 \mathrm{e}-3 \pm 0.00004$ & $16.3 \pm 0.4 \mu \mathrm{M}$ \\
\hline JK-124 & & & $82 \pm 4$ & $3.6 \mathrm{e}-3 \pm 0.0001$ & $44 \pm 2 \mu \mathrm{M}$ \\
\hline JK-125 & $\xi-\mathrm{Cr}$ & & $16 \pm 1$ & $1.4 \mathrm{e}-4 \pm 0.0001$ & $8.7 \pm 6 \mu \mathrm{M}$ \\
\hline
\end{tabular}

B
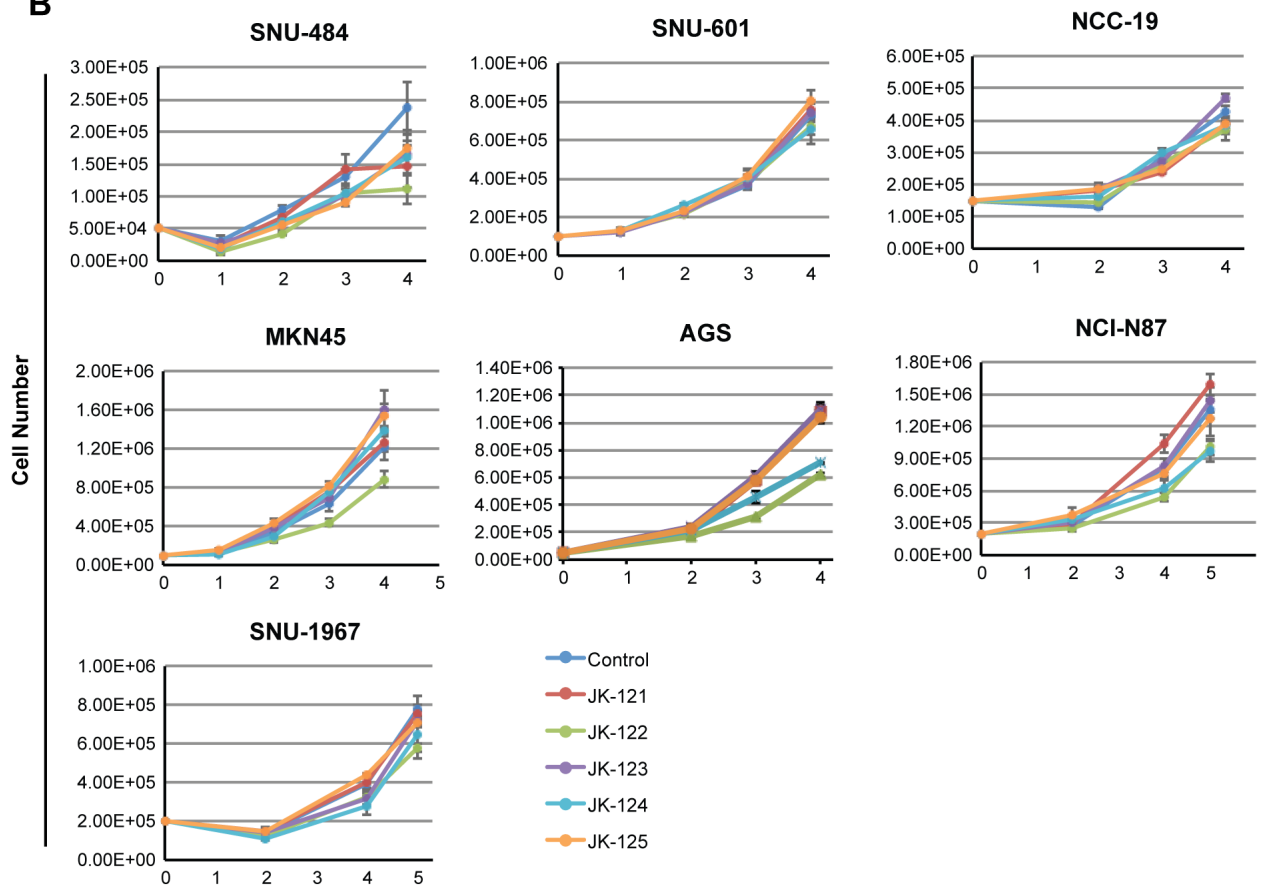

Days

Figure 8: Small molecule inhibitor RHOA candidates inhibit GC cell growth. A. scheme and structure of the five candidates. Reagents and conditions: $\mathrm{MeOH}$ or EtOH, RT or reflux, 2-40 h. On and off rates as well as binding constant $\left(\mathrm{K}_{\mathrm{D}}\right.$ was determined by surface plasmon resonance. B. 7 GC cell lines, SNU-484, SNU-601, NCC-19, MKN-45, AGS, NCI-N87 and SNU-1967 were treated with the five small molecular candidates. GC cell lines showed growth inhibition when treated with $20 \mu \mathrm{M}$ of JK-122. 
was accompanied by a compensatory increase of $R H O B$, inducing apoptosis, as previously shown [24, 25]. However, while $R H O B$ was downregulated in Asian GC tumors, it remained unchanged in Caucasians (Figure 6C; see sample information in Supplementary Table S1). Moreover, the siRHOA-knocked down RHOA low-expressing cell line, SNU-1967, showed minimal growth inhibition, even while RHOB was upregulated (Figure 6B). Consequently, more study is needed to understand the reciprocal relationship between RHOA and RHOB expression.

RHOA is also a known mediator of the epithelialto-mesenchymal transition (EMT) [26, 27]. This process is necessary not only for metastasis, but also for single tumor cell peritoneal dissemination that often occurs in metastatic gastric and ovarian cancers [28, 29]. Functionally, RHOA is a small GTPase, which assumes an active form when bound to GTP, and an inactive form when GTP is hydrolyzed to GDP by GTPase-activating proteins [30]. The active form is then restored by transfer of a phosphate group from guanine nucleotide exchange factors (GEFs). The active GTP-bound RHOA triggers activity of its downstream effectors, including mediators of EMT, upon activation of GEFs by numerous metastasisassociated cytokines such as TGF $\beta$, epidermal growth factor, pro-inflammatory factors, and integrins [27, 3133]. RHOA has also facilitates microtubule remodeling [34], and taken together, these results strongly support the possible efficacy of its therapeutically targeting of the RHOA oncoprotein. To that end, various small molecule RHOA inhibitors are now being examined, including Rhosin and CCG-1423, an inhibitor of RHOA downstream transcription [18]. Here, we also report an innovative strategy for rationally designing RHOA inhibitors.

In summary, we demonstrate involvement of the oncogenic signal mediator RHOA, in gastric cancer, likely via EMT-related cytoskeletal remodeling necessary for cell motility and changes in morphology [31]. This study also supports the importance of pathway construction for developing biomarker-driven cancer therapies. Our analysis clearly shows that RHOA's involvement in GC etiology differs between racial groups, and possibly even between patients. We believe our systematic approach, using distinct cell lines, will greatly contribute to patient stratification, based on RHOA and other pathway genes' expression. In conclusion, our study establishes RHOA inhibition as a potential treatment for Asian GC patients, warranting further investigation with higher statistical power, and assessment of various small molecule RHOA inhibitors, for the therapy of GC.

\section{MATERIALS AND METHODS}

\section{Systems biology analysis}

PATHOME [8] was used to identify statistically significant subpathways (between GC tissues and normal tissues) (p-value cutoff of 0.05 ) from pathways of the entire KEGG [35] signaling network database based on our previously studied Korean GC RNA-Seq dataset [GEO accession: GSE36968] [16]. This dataset consisted of 6 normal gastric tissues and 24 GC tumors, and the network consisted of 559 nodes and 2,031 edges. From that specific network, we inspected all known RHOA-downstream genes, finding 63 that were consistently upregulated in two independent GC patient datasets [GEO accessions: GSE13861, GSE27342] [36, 37].

\section{TCGA GC dataset analysis for Asians and Caucasians}

CBioPortal [38] and the UCSC Cancer Genomics Browser (CGB) [39] were used for analysis. GC and normal sample identifiers for both Asians and Caucasians were obtained from the "TCGA, Nature 2014" data version in CBioPortal [38]. The "TCGA_STAD_exp_ HiSeq-2015-01-28" data version in the UCSC CGB was used to extract gene expression data for both $\mathrm{GC}$ and normal samples for each race.

\section{In silico approach for identifying 730 RHOA small molecule compounds}

For detailed methods, refer to "Supplementary Methods." In brief, based on the structure of the known RHOA inhibitor Rhosin [18], we used Tanimoto similarity scores to search the PUBCHEM database [40], using the program Open Babel [41]. From approximately 45 million compounds, we found 730 similar backbone compounds with high Tanimoto scores of 0.6. Docking software, AutoDock Vina [42] was used, and center of mass (COM) was calculated using calculated using CHARMM [43].

\section{Lipinski's rule application to the 730 compounds}

For detailed methods, refer to "Supplementary Methods." For further details, refer to "Supplementary Methods."

\section{Specific binding test}

The significance of the energy differences for all the candidate compounds was calculated using the statistical method used in DEGseq [44]. For detailed methods, refer to "Supplementary Methods."

\section{Small molecule synthesis}

For detailed methods, refer to "Supplementary Methods."

\section{Cell culture}

The following human GC cell lines were used within 6 months of tissue resuscitation: NCI-N87, AGS (ATCC), 
MKN45 (RIKEN), SNU-484, SNU-601, and SNU-1967 (KCLB), cultured in RPMI-1640 (HyClone) and 10\% fetal calf serum (HyClone) at $37^{\circ} \mathrm{C}$ under $5 \% \mathrm{CO}_{2}$. Cell line identities were validated by short tandem repeat profiling (ATCC, RIKEN, and/or KCLB).

\section{Short hairpin RNA silencing and mouse xenograft $R H O A$-knockdown model}

MISSION ${ }^{\circledR}$ short hairpin RNAs (shRNAs) lentiviral particles (Sigma-Aldrich) were used to stably infect SNU484 and SNU-601 cells (shRNA empty vector or RHOA shRNA), followed by cell culture in 12-well plates using RPMI-1640 plus 10\% FBS, with selection by $0.3 \mathrm{mg} / \mathrm{mL}$ puromycin (Sigma-Aldrich). Western blotting was used to validate decreased RHOA expression, and clones with the least $R H O A$ expression were harvested for injection intomice. Approximately 5-7 x $10^{6}$ cells in log-growth phase were suspended in $0.1 \mathrm{ml}$ phosphate buffered saline (PBS), and subcutaneously injected into the flanks of severe combined immunodeficient (scid) mice (Orient Bio). Animals were weighed weekly and tumor diameters measured twice weekly at right angles $\left(\mathrm{d}_{\text {short }}\right.$ and $\left.\mathrm{d}_{\text {long }}\right)$ with electronic calipers, with conversion to volume by the formula $\mathrm{V}=\left[\left(\mathrm{d}_{\text {long }}\right) \times\left(\mathrm{d}_{\text {short }}\right) \times\left(\mathrm{d}_{\text {long }}\right) / 2\right]$. When the tumors reached volumes reached 150 and $300 \mathrm{~mm}^{3}$, the mice were randomly stratified into two groups of 8 animals, with approximately equal mean tumor volumes.

\section{Small interfering RNA transfection}

$40 \mathrm{nM}$ of anti-RHOA (siRNA-RHOA) small interfering RNA (siRNA) SMARTpools, and nontargeting, scrambled control sequences (Dharmacon/ GE Healthcare), were used to transfect cells, using DharmaFECT1 transfection reagent (Dharmacon/GE Healthcare). Transfection media was replenished with fresh media after $24 \mathrm{~h}$, and the cells then cultured in $5 \% \mathrm{CO}_{2}$ at $37^{\circ} \mathrm{C}$, up to 4 days. Gene knockdown was confirmed by RT-PCR.

\section{Western blot}

Cells were washed twice with $\mathrm{PBS}$, lysed in $20 \mathrm{mM}$ Tris $\mathrm{pH} 7.4,250 \mathrm{mM} \mathrm{NaCl}, 2 \mathrm{mM}$ EDTA, and $1 \%$ Triton X-100 buffer, and centrifuged. Supernatants were collected, total protein concentrations determined using BCA protein assay (Pierce), and subjected to PAGE and immunoblotting using anti-RHOA (ab54835, Abcam), anti- $\beta$-actin (clone 4967, Cell Signaling), and anti- $\alpha$-tubulin (clone 05-829, Millipore) antibodies, in conjunction with anti-rabbit (\#7074S, Cell Signaling) and anti-mouse (\#7076S, Cell Signaling) secondary antibodies. Antibody-bound blots were then visualized by enhanced epichemiluminescence, and quantified using Image Lab software (Bio-Rad).

\section{Cell migration assay}

Radius $^{\text {TM }}$ 24-Well Cell Migration Assay kits (Cell Biolabs) were used according to the manufacturer's protocol. $0.5 \times 10^{5}$ (AGS) or $2.5 \times 10^{5}$ cells (NCI-N87 and SNU-1967) cells were then plated and incubated for a 24-hr attachment. siScri and siRHOA was transfected using DharmaFECTI (Dharmacon/GE Healthcare) and non-treated control. Each condition was done with $n=3$ - 4. After $24 \mathrm{hr}$ incubation, the media was removed, and $500 \mu \mathrm{l}$ fresh media then added to each well. Picturess were taken at 0, 5 and $8 \mathrm{hr}$ intervals, using an Olympus IX70 microscope under brightfield. Migration was observed until about 50\% wound closure (AGS: 12 hr; NCI-N87: $28 \mathrm{hr}$ and SNU-1967: $30 \mathrm{hr}$ ), and at endpoint, pictures were taken after cells were stained using Cell Staining Solution. Migration edges and wound area was analyzed using Image $\mathrm{J}(\mathrm{NIH})$ and Cell Profiler (Broad Institute). $\%$ wound closure was calculated by measuring the wound area at $0 \mathrm{hr}\left(\mathrm{A}_{0}\right)$, and measuring the wound area at the endpoint $\left(A_{T}\right)$, and calculating $\left(A_{0}-A_{T}\right) \times 100 \%$.

\section{Real time-PCR}

Total RNA was isolated from cell lysates using Isol-RNA Lysis Reagent (5Prime). After lysis, $0.2 \mathrm{ml}$ chloroform was added, the samples shaken for 15 seconds, and then centrifuged at $13,000 \mathrm{rpm}$ for $15 \mathrm{~min}$ at $4^{\circ} \mathrm{C}$. After centrifugation, the upper layers were placed into new tubes, and $0.5 \mathrm{ml}$ isopropanol was added. The samples were mixed gently, incubated at RT for 10 minutes, and re-centrifuged at $13 \times 1000 \mathrm{rpm}$ for 10 minutes at $4^{\circ} \mathrm{C}$. Supernatants were removed and $1 \mathrm{ml}$ of $70 \% \mathrm{EtOH}$ were added to the pellets, followed by centrifugation at $7500 \mathrm{rpm}$ for 5 minutes at $4^{\circ} \mathrm{C}$. The RNA pellets were then dried and dissolved in DEPC-treated water. cDNA was synthesized using ReverTra Ace ${ }^{\circledR}$ qPCR RT Master Mix with gDNA Remover kits (Toyobo). RT-PCR was performed using a CFX384 system (Bio-Rad) and iQ ${ }^{\mathrm{TM}}$ SYBR ${ }^{\circledR}$ Green Supermix (Bio-Rad) using primers designed by Primer-BLAST (http://www.ncbi.nlm.nih. gov/tools/primer-blast/) or GenScript (www.genscript. com). Relative expression levels were normalized to GAPDH of the siScr sample corresponding to the day of sampling, using the 2(-delta-delta $\mathrm{C}_{\mathrm{T}}$ ) method [45]. All measurements were performed in triplicate.

\section{Cell cycle analysis by flow cytometry}

Negative control cells and cells transfected with siRNA-non-targeting sequences or siRNA-RHOA were harvested on days 2, 3 and 4. Collected cells were centrifuged at $1200 \mathrm{rpm}$ for 10 minutes, and washed with $10 \mathrm{ml}$ PBS. After centrifuging, PBS was removed and cold $80 \%$ EtOH was added drop-wise to the well-suspended cells. After centrifugation and removal of EtOH, the cells 
were incubated in $1 \mathrm{ml}$ propidium iodide (PI; $50 \mu \mathrm{g} /$ $\mathrm{ml})$ with RNase A $(0.1 \mathrm{mg} / \mathrm{ml})$ for 30 minutes at $37^{\circ} \mathrm{C}$, centrifuged to remove the unbound PI, and resuspended in PBS for cell cycle analysis using a FACS Calibur (BD Biosciences) flow cytometer.

\section{RHOA binding of small molecule candidate inhibitors}

Surface Plasmon Resonance (SPR) was used to study the binding of RHOA protein to synthesized small molecules. Reichert SR7500DC system was used, and RHOA (SRP5127, Sigma Aldrich) protein was immobilized on CMDH gold chip (Reichert) at $<7 \mu \mathrm{g}$ and a flow rate of $10 \mu \mathrm{l} / \mathrm{min}$. Rhosin (Millipore) and compounds JK-121 125 were dissolved in DMSO. Immobilized RHOA resulted in 2550 resonance units (RU). CLAMP ${ }^{\circ}$ program [46] was used to analyzed the kinetics of protein-small molecule binding.

\section{CONFLICTS OF INTEREST}

The authors declare no conflicts of interest.

\section{GRANT SUPPORT}

This study was supported by grants from the National Cancer Center of the Republic of Korea, NCC-1210350-3, NCC-1510500-1 National Research Foundation of Korea (MSIP) 2015R1A2A1A10052661 (to YHK); National Research Foundation of Korea 2015R1D1A1A01059381 (HRC); National Cancer Center of the Republic of Korea, NCC-1210460, NCC-1510140-1, the Gachon University Gil Medical Center (Grant number: 2016-06) (to SN); Bio-Synergy Research Project (2013M3A9C4078158) of the Ministry of Science, ICT and Future Planning through the National Research Foundation and the Korea Health Technology R\&D Project through the Korea Health Industry Development Institute (KHIDI), funded by the Ministry of Health \& Welfare, Republic of Korea (HI15C2165) (to TP); and from the Korean Research Institute of Bioscience and the Biotechnology Research Initiative Program (to JL).

\section{REFERENCES}

1. International Agency for Cancer Research, WHO, GLOBOCAN 2012: Estimated Cancer Incidence, Mortality and Prevalence Worldwide.

2. Bang YJ, Kim YW, Yang HK, Chung HC, Park YK, Lee KH, Lee KW, Kim YH, Noh SI, Cho JY, Mok YJ. Adjuvant capecitabine and oxaliplatin for gastric cancer after D2 gastrectomy (CLASSIC): a phase 3 open-label, randomised controlled trial. The Lancet. 2012; 379:315-321.
3. Roukos DH. Current status and future perspectives in gastric cancer management. Cancer Treat Rev. 2000; 26:243-255.

4. Hartgrink HH, Jansen EP, van Grieken NC, van de Velde CJ. Gastric cancer. Lancet. 2009; 374:477-490.

5. Bang YJ, Van Cutsem E, Feyereislova A, Chung HC, Shen L, Sawaki A, Lordick F, Ohtsu A, Omuro Y, Satoh T, Aprile G, Kulikov E, Hill J, Lehle M, Ruschoff J, Kang YK, et al. Trastuzumab in combination with chemotherapy versus chemotherapy alone for treatment of HER2-positive advanced gastric or gastro-oesophageal junction cancer (ToGA): a phase 3, open-label, randomised controlled trial. Lancet. 2010; 376:687-697.

6. Takeno A, Takemasa I, Seno S, Yamasaki M, Motoori M, Miyata H, Nakajima K, Takiguchi S, Fujiwara Y, Nishida T, Okayama T, Matsubara K, Takenaka Y, Matsuda H, Monden M, Mori M, et al. Gene expression profile prospectively predicts peritoneal relapse after curative surgery of gastric cancer. Ann Surg Oncol. 2010; 17:1033-1042.

7. Barabasi AL, Gulbahce N, Loscalzo J. Network medicine: a network-based approach to human disease. Nat Rev Genet. 2011; 12:56-68.

8. Nam S, Chang HR, Kim KT, Kook MC, Hong D, Kwon CH, Jung HR, Park HS, Powis G, Liang H, Park T, Kim YH. PATHOME: an algorithm for accurately detecting differentially expressed subpathways. Oncogene. 2014; 33:4941-4951.

9. Nam S, Chang HR, Jung HR, Gim Y, Kim NY, Grailhe R, Seo HR, Park HS, Balch C, Lee J, Park I, Jung SY, Jeong KC, Powis G, Liang H, Lee ES, et al. A pathway-based approach for identifying biomarkers of tumor progression to trastuzumab-resistant breast cancer. Cancer Lett. 2015; 356:880-890.

10. Chang HR, Nam S, Kook MC, Kim KT, Liu X, Yao H, Jung HR, Lemos R, Jr., Seo HH, Park HS, Gim Y, Hong D, Huh I, Kim YW, Tan D, Liu CG, et al. HNF4alpha is a therapeutic target that links AMPK to WNT signalling in early-stage gastric cancer. Gut. 2016; 65:19-32.

11. Taniguchi K, Shunichi Tsujitani, Naruo Tokuyasu, Takuji Naka, Shigeru Tatebe, Akira Kondo, Ikeguchi M. RhoROCK expression predicts the prognosis in patients with T3/T4 gastric cancer. Yonago Acta medica. 2007; 50:9-15.

12. Pan Y, Bi F, Liu N, Xue Y, Yao X, Zheng Y, Fan D. Expression of seven main Rho family members in gastric carcinoma. Biochem Biophys Res Commun. 2004; 315:686-691.

13. The Cancer Genome Atlas Network. Comprehensive molecular characterization of gastric adenocarcinoma. Nature. 2014; 513:202-209.

14. Kakiuchi M, Nishizawa T, Ueda H, Gotoh K, Tanaka A, Hayashi A, Yamamoto S, Tatsuno K, Katoh H, Watanabe Y, Ichimura T, Ushiku T, Funahashi S, Tateishi K, Wada I, Shimizu N, et al. Recurrent gain-of-function mutations of RHOA in diffuse-type gastric carcinoma. Nat Genet. 2014; 46:583-587. 
15. Wang K, Yuen ST, Xu J, Lee SP, Yan HH, Shi ST, Siu HC, Deng S, Chu KM, Law S, Chan KH, Chan AS, Tsui WY, Ho SL, Chan AK, Man JL, et al. Whole-genome sequencing and comprehensive molecular profiling identify new driver mutations in gastric cancer. Nat Genet. 2014; 46:573-582.

16. Kim YH, Liang H, Liu X, Lee JS, Cho JY, Cheong JH, Kim H, Li M, Downey TJ, Dyer MD, Sun Y, Sun J, Beasley EM, Chung HC, Noh SH, Weinstein JN, et al. AMPKalpha modulation in cancer progression: multilayer integrative analysis of the whole transcriptome in Asian gastric cancer. Cancer Res. 2012; 72:2512-2521.

17. Kim S, Thiessen PA, Bolton EE, Chen J, Fu G, Gindulyte A, Han L, He J, He S, Shoemaker BA, Wang J, Yu B, Zhang J, Bryant SH. PubChem Substance and Compound databases. Nucleic Acids Res. 2016; 44:D1202-1213.

18. Shang X, Marchioni F, Sipes N, Evelyn CR, JerabekWillemsen M, Duhr S, Seibel W, Wortman M, Zheng Y. Rational design of small molecule inhibitors targeting RhoA subfamily Rho GTPases. Chem Biol. 2012; 19:699-710.

19. Lipinski CA. Lead- and drug-like compounds: the rule-offive revolution. Drug discovery today Technologies. 2004; $1: 337-341$.

20. Kristelly R, Gao G, Tesmer JJ. Structural determinants of RhoA binding and nucleotide exchange in leukemiaassociated Rho guanine-nucleotide exchange factor. J Biol Chem. 2004; 279:47352-47362.

21. Strong VE, Song KY, Park CH, Jacks LM, Gonen M, Shah MA, Coit DG, Brennan MF. Comparison of disease-specific survival in the United States and Korea after resection for early-stage node-negative gastric carcinoma. J Surg Oncol. 2013; 107:634-640.

22. Wainberg ZA, Anghel A, Desai AJ, Ayala R, Luo T, Safran B, Fejzo MS, Hecht JR, Slamon DJ, Finn RS. Lapatinib, a dual EGFR and HER2 kinase inhibitor, selectively inhibits HER2-amplified human gastric cancer cells and is synergistic with trastuzumab in vitro and in vivo. Clin Cancer Res. 2010; 16:1509-1519.

23. Sharma SV, Haber DA, Settleman J. Cell line-based platforms to evaluate the therapeutic efficacy of candidate anticancer agents. Nat Rev Cancer. 2010; 10:241-253.

24. Zhou J, Zhu Y, Zhang G, Liu N, Sun L, Liu M, Qiu M, Luo D, Tang Q, Liao Z, Zheng Y, Bi F. A distinct role of RhoB in gastric cancer suppression. Int $\mathrm{J}$ Cancer. 2011; 128:1057-1068.

25. Prendergast GC. Actin' up: RhoB in cancer and apoptosis. Nat Rev Cancer. 2001; 1:162-168.

26. Nguyen DX, Bos PD, Massague J. Metastasis: from dissemination to organ-specific colonization. Nat Rev Cancer. 2009; 9:274-284.

27. Shankar J, Nabi IR. Actin cytoskeleton regulation of epithelial mesenchymal transition in metastatic cancer cells. PLoS One. 2015; 10:e119954.

28. Yonemura Y, Bandou E, Kawamura T, Endou Y, Sasaki T. Quantitative prognostic indicators of peritoneal dissemination of gastric cancer. Eur J Surg Oncol. 2006; 32:602-606.

29. Yawata A, Adachi M, Okuda H, Naishiro Y, Takamura T, Hareyama M, Takayama S, Reed JC, Imai K. Prolonged cell survival enhances peritoneal dissemination of gastric cancer cells. Oncogene. 1998; 16:2681-2686.

30. Ren XD, Kiosses WB, Schwartz MA. Regulation of the small GTP-binding protein Rho by cell adhesion and the cytoskeleton. EMBO J. 1999; 18:578-585.

31. Matsuoka T, Yashiro M. Rho/ROCK signaling in motility and metastasis of gastric cancer. World J Gastroenterol. 2014; 20:13756-13766.

32. Bhowmick NA, Ghiassi M, Bakin A, Aakre M, Lundquist CA, Engel ME, Arteaga CL, Moses HL. Transforming growth factor-betal mediates epithelial to mesenchymal transdifferentiation through a RhoA-dependent mechanism. Mol Biol Cell. 2001; 12:27-36.

33. Thiery JP, Sleeman JP. Complex networks orchestrate epithelial-mesenchymal transitions. Nat Rev Mol Cell Biol. 2006; 7:131-142.

34. Nakaya Y, Sukowati EW, Wu Y, Sheng G. RhoA and microtubule dynamics control cell-basement membrane interaction in EMT during gastrulation. Nat Cell Biol. 2008; 10:765-775.

35. Kanehisa M. The KEGG database. Novartis Found Symp. 2002; 247:91-101; discussion 101-103, 119-128, 244-152.

36. Cho JY, Lim JY, Cheong JH, Park YY, Yoon SL, Kim SM, Kim SB, Kim H, Hong SW, Park YN, Noh SH, Park ES, Chu IS, Hong WK, Ajani JA, Lee JS. Gene expression signature-based prognostic risk score in gastric cancer. Clin Cancer Res. 2011; 17:1850-1857.

37. Cui J, Li F, Wang G, Fang X, Puett JD, Xu Y. Geneexpression signatures can distinguish gastric cancer grades and stages. PLoS One. 2011; 6:e17819.

38. Cerami E, Gao J, Dogrusoz U, Gross BE, Sumer SO, Aksoy BA, Jacobsen A, Byrne CJ, Heuer ML, Larsson E, Antipin Y, Reva B, Goldberg AP, Sander C, Schultz N. The cBio cancer genomics portal: an open platform for exploring multidimensional cancer genomics data. Cancer Discov. 2012; 2:401-404.

39. Goldman M, Craft B, Swatloski T, Ellrott K, Cline M, Diekhans M, Ma S, Wilks C, Stuart J, Haussler D, Zhu J. The UCSC Cancer Genomics Browser: update 2013. Nucleic Acids Res. 2013; 41:D949-954.

40. Shang S, Tan DS. Advancing chemistry and biology through diversity-oriented synthesis of natural product-like libraries. Curr Opin Chem Biol. 2005; 9:248-258.

41. O'Boyle NM, Banck M, James CA, Morley C, Vandermeersch T, Hutchison GR. Open Babel: An open chemical toolbox. J Cheminform. 2011; 3:33.

42. Trott O, Olson AJ. AutoDock Vina: improving the speed and accuracy of docking with a new scoring function, efficient optimization, and multithreading. J Comput Chem. 2010; 31:455-461. 
43. Brooks BR, Brooks CL, 3rd, Mackerell AD, Jr., Nilsson L, Petrella RJ, Roux B, Won Y, Archontis G, Bartels C, Boresch S, Caflisch A, Caves L, Cui Q, Dinner AR, Feig M, Fischer S, et al. CHARMM: the biomolecular simulation program. J Comput Chem. 2009; 30:1545-1614.

44. Wang L, Feng Z, Wang X, Wang X, Zhang X. DEGseq: an $\mathrm{R}$ package for identifying differentially expressed genes from RNA-seq data. Bioinformatics. 2010; 26:136-138.
45. Livak KJ, Schmittgen TD. Analysis of relative gene expression data using real-time quantitative PCR and the 2(-Delta Delta C(T)) Method. Methods. 2001; 25:402-408.

46. Myszka DG, Morton TA. CLAMP: a biosensor kinetic data analysis program. Trends Biochem Sci. 1998; 23:149-150. 